\title{
Propagação vegetativa de liamba, planta medicinal
}

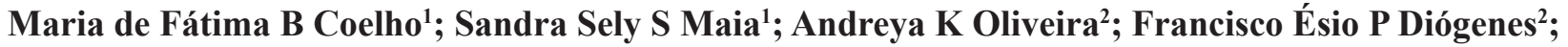 \\ Silvio Roberto F Soares ${ }^{2}$ \\ ${ }^{1}$ UFERSA, Pós-Graduação em Fitotecnia, C. Postal 137, 59625-900 Mossoró-RN; ${ }^{2}$ UFERSA, Depto. Ciências Vegetais, Progr. Iniciação \\ Cientifica PIBIC/CNPq; coelhomfstrela@gmail.com
}

\section{RESUMO}

A liamba (Vitex agnus castus L.) é utilizada no Brasil como medicinal e apresenta compostos com estrutura química semelhante à progesterona. Visando avaliar a propagação vegetativa de liamba foram conduzidos dois experimentos. O primeiro, com estacas da parte aérea com $20 \mathrm{~cm}$ de comprimento, sem folhas e classificadas em dois grupos: apical e basal. As estacas tiveram a espessura padronizada em 3 a 5 mm de diâmetro e 40 unidades de cada tipo foram colocadas em sacolas de polietileno $(10 \times 30 \mathrm{~cm})$ preenchidas com o substrato solo + esterco na proporção volumétrica de 2:1. O segundo, com miniestacas da parte aérea da planta com 3 a $5 \mathrm{~cm}$ de comprimento, sem folhas e no delineamento experimental em blocos casualizados com quatro repetições de 20 miniestacas, no esquema fatorial 2 × 3 . Os tratamentos foram tipo da miniestaca (com talão e sem talão) e substrato (solo; solo + esterco bovino curtido e solo + composto). A propagação vegetativa de liamba pode ser feita por estacas basais ou mini-estacas com talão em substrato solo + composto.

Palavras-chave: Vitex agnus castus, substrato, estaca basal, miniestacas, planta medicinal.

\begin{abstract}
Vegetative propagation of liamba, a medicinal plant

The liamba (Vitex agnus castus L.) is used as a medicinal species in Brazil and presents compounds with similar chemical structure to progesterone. To evaluate the propagation of liamba two experiments were carried out. The first, using shoot cuttings with $20 \mathrm{~cm}$ long, leafless and classified into two groups, apical and basal. The cuttings were standardized in their thickness of 3 to $5 \mathrm{~mm}$ in diameter and 40 units of each type were placed in polyethylene bags $(10 \times 30 \mathrm{~cm})$ filled with soil + manure in the volumetric ratio of $2: 1$. The second, with shoot minicuttings of the plant canopy with 3 to $5 \mathrm{~cm}$ long, without leaves and in a randomized block experimental design with four replications of 20 minicuttings, in a factorial $2 \times 3$. The treatments were kind of mini-cuttings (with and without hell) and substrate (soil, soil + cattle manure and soil + compost). The vegetative propagation of liamba can be made by basal cuttings or minicuttings with heel in soil + compost.
\end{abstract}

Keywords: Vitex agnus castus, substrate, basal cuttings, minicuttings, medicinal plant.

\section{(Recebido para publicação em 17 de agosto de 2010; aceito em 21 de agosto de 2011)}

(Received on August 17, 2010; accepted on August 21, 2011)

\begin{abstract}
$\mathrm{A}$ liamba (Vitex agnus castus L.), pertence à família Verbenaceae, ordem Lamiales, ocorre no Mediterrâneo e na Criméia sendo encontrada também em regiões quentes da Ásia, África e Américas. É conhecida no Brasil como liamba ou quiabo de Angola, tem sido utilizada popularmente como medicinal e em propósitos sociais e religiosos pelos descendentes culturais dos africanos, ou seja, comunidades religiosas que conservam o conhecimento tradicional das populações negras que chegaram ao Brasil como escravas (Maia et al., 2001). Os frutos e as folhas são usados no tratamento da TPM (tensão pré-menstrual), menopausa e como anafrodisíaco (Matos, 2002). A espécie já conta com estudos farmacológicos que mostraram a ação normalizadora e balanceadora (estrógeno-progesterona) benéfica no tratamento de menstruação irregular e dolorosa, infertilidade, síndrome pré-menstrual, problemas de menopausa e
\end{abstract}

outros desequilíbrios hormonais, sendo útil no tratamento de endometriose e também para normalizar o sistema hormonal após o uso descontinuado de pílulas anticoncepcionais (Schellenberg, 2001; Veal, 1998; Sliutz et al., 1993).

A espécie está bem adaptada em várias regiões brasileiras, mas as sementes apresentam dormência (Belhadj et al., 1998) e a propagação por estacas é importante por manter as características genéticas das plantas selecionadas, reduzir o porte e o ciclo vegetativo. A estaquia é a técnica de propagação mais fácil que vem sendo muito utilizada nas espécies que apresentam facilidade em formar raízes adventícias (Hartmann et al., 2002). A técnica de estaquia consiste na multiplicação de plantas com uso de segmentos de ramos ou raízes, providos de gemas meristemáticas com capacidade para emitir raízes adventícias, comumente denominadas estacas (Hartmann et al., 2002).
Em árvores, de acordo com a menor ou maior lignificação dos tecidos, pode-se dispor de estacas lenhosas, semilenhosas e herbáceas e segundo Hartmann et al. (2002) as estacas herbáceas e semilenhosas geralmente enraízam com maior facilidade e rapidez que as lenhosas, pois a menor lignificação dos tecidos facilita a passagem das raízes formadas no periciclo.

A propagação vegetativa por estaquia foi utilizada de forma eficiente na multiplicação de diversas espécies medicinais, tais como bamburral (Hyptis suaveolens) (Maia et al., 2008); carqueja (Baccharis trimera (Less.) DC.) (Carvalho et al., 2007); hortelã-japonesa (Mentha arvensis L.) (Chagas et al., 2008); espinheira-santa (Maytenus ilicifolia) (Lima et al., 2008); atroveran (Ocimum selloi Benth.) (Costa et al., 2007) e crajiru (Arrabidaea chica (Humb. \& Bonpl.) B. Verl.) (Ferreira \& Gonçalves, 2007). 
Estudar os fatores inerentes à estaca, como por exemplo, a posição da estaca no ramo, o grau de lignificação, a quantidade de reservas, a diferenciação dos tecidos é muito importante, pois segundo Lima et al. (2006), a escolha do ramo e a posição da retirada da estaca são fatores que induzem grande variação no desenvolvimento de raízes.

Objetivou-se com a realização desse trabalho, verificar o melhor tipo de estaca e substrato para a propagação vegetativa da liamba.

\section{MATERIAL E MÉTODOS}

Os experimentos foram conduzidos de março a junho de 2007, em casa de vegetação da UFERSA, no município de Mossoró-RN $\left(5^{\circ} 11^{\prime} \mathrm{S}, 37^{\circ} \mathrm{W}\right.$, altitude média de 18 metros). Segundo a classificação de Köppen, o clima é do tipo BSwh', apresentando temperatura média anual de $27,4^{\circ} \mathrm{C}$, precipitação pluviométrica bastante irregular, com média de 673,9 mm/ano e umidade relativa média de 68,9\% (Carmo Filho et al., 1991).

Foram obtidas estacas da parte aérea da planta adulta no estádio de florescimento, localizada no Horto de Plantas Medicinais da UFERSA. No primeiro experimento utilizou-se estacas semi-lenhosas sem folhas com $20 \mathrm{~cm}$ de comprimento e espessura padronizada em 3 a 5 mm de diâmetro. As estacas foram classificadas quanto a sua posição no ramo em dois grupos apical e basal, os quais constituíram os tratamentos. Foram utilizadas quatro repetições de 10 estacas, colocadas em sacos de polietileno $(10 \times 30 \mathrm{~cm})$ preenchidas com o substrato arisco (latossolo utilizado em viveiros de Mossoró semelhante ao terriço do Sul) + esterco bovino curtido na proporção de 2:1. As irrigações foram feitas com regador no inicio da manhã e final da tarde, e cada estaca foi protegida com sacola de polietileno transparente até a emissão das brotações. Aos 90 dias foram avaliadas as características: número de brotações por estaca, número de folhas por estaca, comprimento da maior raiz $(\mathrm{cm})$, massa seca total por estaca (g) e a porcentagem de sobrevivência. A análise estatística foi efetuada no SAEG, pelo teste t a $5 \%$ de probabi- lidade (Ribeiro Junior \& Melo, 2009).

No segundo experimento foram obtidas, na mesma árvore do primeiro $\mathrm{cm}$ de comprimento e sem folhas. $\mathrm{O}$ delineamento experimental foi em blocos ao acaso com quatro repetições de 20 miniestacas, no esquema fatorial $2 \times 3$. Os níveis dos fatores foram tipo substrato [solo; solo + esterco bovino curtido (2:1) e solo + composto (2:1)]. As miniestacas foram colocadas em bandejas de poliestireno expandido de 128 células de $5 \mathrm{~cm}$ de profundidade preenchidas com os substratos. As miniestacas com talão diferenciam-se das sem talão por trazer parte do lenho velho, pois foram obtidas destacando-se um ramo no ponto de inserção com outro ramo de dois anos. As características químicas e físicas dos substratos utilizados foram: Solo $-\mathrm{pH}=5,8, \mathrm{P}=$ $1,9 \mathrm{mg} \mathrm{dm}^{-3}, \mathrm{~K}=26,2 \mathrm{mg} \mathrm{dm}{ }^{-3}, \mathrm{Na}=$ $16,7 \mathrm{mg} \mathrm{dm}^{-3}, \mathrm{Ca}=1,8 \mathrm{cmol}_{\mathrm{c}} \mathrm{dm}^{-3}, \mathrm{Mg}=$ $2,4 \mathrm{cmol}_{\mathrm{c}} \mathrm{dm}^{-3}, \mathrm{Al}=0,05 \mathrm{cmol}_{\mathrm{c}} \mathrm{dm}^{-3}$, $\mathrm{H}+\mathrm{Al}=0,5 \mathrm{cmol}_{\mathrm{c}} \mathrm{dm}^{-3}, \mathrm{SB}=4,34 \mathrm{cmol}_{\mathrm{c}}$ $\mathrm{dm}^{-3}, \mathrm{CTC}=4,83^{\mathrm{c}} \mathrm{cmol}_{\mathrm{c}} \mathrm{dm}^{-3}$, densidade aparente $=1,36 \mathrm{~g} / \mathrm{cm}^{3}$, densidade real $=$ $2,60 \mathrm{~g} / \mathrm{cm}^{3}$, porosidade total $=47,53 \%$, argila $=15$, silte $=9$, areia $=76 \%$; Solo + esterco - $\mathrm{pH}=7,1, \mathrm{P}=65,9 \mathrm{mg} \mathrm{dm}^{-3}$, $\mathrm{K}=550,1 \mathrm{mg} \mathrm{dm}^{-3}, \mathrm{Na}=127 \mathrm{mg} \mathrm{dm}^{-3}$, $\mathrm{Ca}=2,1 \mathrm{cmol}_{\mathrm{c}} \mathrm{dm}^{-3}, \mathrm{Mg}=2,9 \mathrm{cmol}_{\mathrm{c}}$ $\mathrm{dm}^{-3}, \mathrm{Al}=0 \mathrm{cmol} \mathrm{dm}^{-3}, \mathrm{H}+\mathrm{Al}=0 \mathrm{cmol}^{-}$ $\mathrm{dm}^{-3}, \mathrm{SB}=6,06 \mathrm{cmol}_{\mathrm{c}} \mathrm{dm}^{-3}, \mathrm{CTC}=6,56$ $\mathrm{cmol}_{\mathrm{c}} \mathrm{dm}^{-3}$, densidade aparente $=1,15 \mathrm{~g} /$ $\mathrm{cm}^{3}$, densidade real $=2,56 \mathrm{~g} / \mathrm{cm}^{3}$, porosidade total $=55,08 \%$, argila $=9$, silte $=$ 8 , areia $=83 \%$; Solo + composto $-\mathrm{pH}=$ $6,1, \mathrm{P}=55,7 \mathrm{mg} \mathrm{dm}^{-3}, \mathrm{~K}=67,2 \mathrm{mg} \mathrm{dm}^{-3}$, $\mathrm{Na}=19,6 \mathrm{mg} \mathrm{dm}^{-3}, \mathrm{Ca}=2,2 \mathrm{cmol}_{\mathrm{c}} \mathrm{dm}^{-3}$, $\mathrm{Mg}=2,7 \mathrm{cmol}_{\mathrm{c}} \mathrm{dm}^{-3}, \mathrm{Al}=0 \mathrm{cmol}_{\mathrm{c}} \mathrm{dm}^{-3}$, $\mathrm{H}+\mathrm{Al}=0 \mathrm{cmol}_{\mathrm{c}} \mathrm{dm}^{-3}, \mathrm{SB}=5,15 \mathrm{cmol}_{\mathrm{c}}$ experimento, miniestacas com 3 a 5 da miniestaca (com talão e sem talão) e

$\mathrm{dm}^{-3}, \mathrm{CTC}=5,43 \mathrm{cmol}_{\mathrm{c}} \mathrm{dm}^{-3}$, densidade aparente $=1,10 \mathrm{~g} / \mathrm{cm}^{3}$, densidade real $=$ $2,18 \mathrm{~g} / \mathrm{cm}^{3}$, porosidade total $=68,90 \%$, argila $=5$, silte $=8$, areia $=87 \%$.

As irrigações foram feitas com regador, quando necessárias, e cada estaca foi protegida com sacola de polietileno transparente até a emissão das brotações. Os dados obtidos foram analisados no SAEG, e as médias comparadas pelo teste Tukey a $5 \%$ de probabilidade, de acordo com Ribeiro Junior \& Melo (2009).

\section{RESULTADOS E DISCUSSÃO}

Em relação ao primeiro experimento verifica-se que as estacas basais apresentaram maior porcentagem de sobrevivência $(60 \%)$, número de brotações $(2,75)$, número de folhas $(30,5)$, comprimento de raiz $(21,0 \mathrm{~cm})$ e maior massa seca total $(4,94 \mathrm{~g})$ que as estacas apicais (Tabela 1). Ao longo do ramo, o conteúdo de carboidratos e de substâncias promotoras e inibidoras do enraizamento apresenta bastante variação e, normalmente, os maiores teores de carboidratos se encontram na base dos ramos contribuindo para o enraizamento e sobrevivência das estacas (Fachinello et al., 2005). Resultados semelhantes foram observados em Sebastiania schottiana (Frasseto, 2008), hortênsia (Luz et al., 2007) e em Ocimum gratissimum (Sousa et. al., 2005).

No segundo experimento, as miniestacas com talão e no substrato solo + composto apresentaram maior percentagem de sobrevivência $(31,25 \%)$, que as miniestacas sem talão nos três substratos. A porcentagem de estacas com brotações não variou significativamente

Tabela 1. Características avaliadas nas estacas apicais e basais de liamba no primeiro experimento (characteristics evaluated on liamba apical and basal cuttings in the first experiment). Mossoró, UFERSA, 2007.

\begin{tabular}{lcc}
\hline Características & Estacas apicais & Estacas basais \\
\hline Número médio de brotações & $2,0 \mathrm{~b}$ & $2,7 \mathrm{a}$ \\
Número médio de folhas & $27,5 \mathrm{~b}$ & $30,5 \mathrm{a}$ \\
Comprimento de raiz $(\mathrm{cm})$ & $5,7 \mathrm{~b}$ & $21,0 \mathrm{a}$ \\
Massa seca total $(\mathrm{g})$ & $3,8 \mathrm{~b}$ & $4,9 \mathrm{a}$ \\
Sobrevivência $(\%)$ & $25,0 \mathrm{~b}$ & $60,0 \mathrm{a}$ \\
\hline
\end{tabular}

Medias seguidas da mesma letra minúsculas na linha não diferem entre si (teste $t \mathrm{p} \leq 0.05$ ) (means followed by the same small letters in the columns did not differ from each other $(\mathrm{t}$ test $\mathrm{p} \leq 0.05)$. 
Tabela 2. Características avaliadas nas miniestacas com e sem talão de liamba no segundo experimento (characteristics evaluated on liamba minicuttings with hell and without hell in the second experiment). Mossoró, UFERSA, 2007.

\begin{tabular}{llccc}
\hline \multirow{2}{*}{ Tipo de estaca } & \multicolumn{3}{c}{ Substratos } \\
\cline { 2 - 5 } & \%olo & Solo + esterco & Solo + composto \\
\hline \multirow{2}{*}{ Com talão } & \% de sobrevivência & $15,6 \mathrm{bA}$ & $3,1 \mathrm{cA}$ & $31,2 \mathrm{aA}$ \\
& $\%$ de enraizamento & $70,3 \mathrm{bA}$ & $50,5 \mathrm{cB}$ & $90,4 \mathrm{aA}$ \\
\hline \multirow{2}{*}{ Sem talão } & $93,7 \mathrm{aA}$ & $96,9 \mathrm{aA}$ & $87,5 \mathrm{aA}$ \\
& $\%$ de brotações & $6,2 \mathrm{bB}$ & $3,1 \mathrm{aA}$ & $0,0 \mathrm{aB}$ \\
& $\%$ de enraizamento & $50,7 \mathrm{aB}$ & $30,0 \mathrm{aB}$ & $40,0 \mathrm{aB}$ \\
\hline
\end{tabular}

As letras minúsculas comparam médias na linha e as maiúsculas médias na coluna, para cada característica (Tukey $\mathrm{p} \leq 0,05$ ) (means followed by the same small letters in the columns and the capital letters in the lines, did not differ from each other (Tukey test $\mathrm{p} \leq 0.05$ ).

entre os substratos, mas a porcentagem de estacas enraizadas foi maior em estacas com talão e no substrato solo + composto (Tabela 2). Este substrato deve ter proporcionado maior aeração, como também constatado por Schmitz et al. (2002), ao avaliar as características físicas de diversos substratos e, dos materiais minerais como solo e areia, que possuem excessiva densidade e reduzida porosidade, sendo deficientes em aeração.

O substrato destinado à produção de mudas deve apresentar boa porosidade, a fim de assegurar boa drenagem e conseqüentemente arejamento do sistema radicular. O substrato solo + composto teve maior porosidade e menor densidade, o que pode ter sido responsável pelo melhor desempenho das mini-estacas de liamba. Isso está de acordo com Arruda et al. (2007), que verificaram que a utilização de composto orgânico, proporcionou a maior porcentagem de enraizamento de estacas de guaranazeiro, assim como Silva et al. (2010) observaram a influência positiva do composto orgânico no enraizamento de estacas de aceroleira favorecendo o desenvolvimento de mudas vigorosas para o plantio. Resultados semelhantes foram obtidos por Pereira et al. (2002) que observaram que o composto orgânico mostrou ser o melhor componente para formulação de substrato para propagação de Coffea arabica por estaquia, concordando com Hoffmann et al. (1995), que obtiveram melhor enraizamento de estacas de mirtilo utilizando o substrato composto por areia + composto orgânico.
Conclui-se, portanto, que as estacas basais com espessura de aproximadamente $5 \mathrm{~mm}$ e comprimento de $20 \mathrm{~cm}$, e as miniestacas com talão no substrato solo + composto podem ser usadas para a propagação vegetativa de liamba.

\section{REFERÊNCIAS}

ARRUDA MR; PEREIRA JCR; MOREIRA A; TEIXEIRA WG. 2007. Enraizamento de estacas herbáceas de guaranazeiro em diferentes substratos. Ciência e Agrotecnologia 31: 236-241.

BELHADJ S; GERASOPOULOS D; MALOUPA E. 1998. Improvement of germination of Vitex agnus castus 1 . seeds with seed pre-treatments. Acta Horticulturae 454: 207-212.

CARMO FILHO F; OLIVEIRA OF 1989. Um município do semi-árido nordestino: características climáticas; aspectos florestais. Mossoró: ESAM, 62p. (Coleção Mossoroense).

CARVALHO RIN; NOLASCO MA; CARVALHO T; RIPKA M; GIUBLIN LM; NEGRELLO M; SCHEFFER MC. 2007. Enraizamento de estacas de carqueja em função de diferentes. Scientia Agraria 8: 269-274.

CHAGAS JH; PINTO JEBP; BERTOLUCCI SKV; NALON FH. 2008. Produção de mudas de hortelã-japonesa em função da idade e de diferentes tipos de estaca. Ciência Rural 38: 2157-2163.

COSTA LCB; PINTO JEBP; BERTOLUCCI SKV. 2007. Comprimento da estaca e tipo de substrato na propagação vegetativa de atroveran. Ciência Rural 37: 1157-1160.

FACHINELLO JC; HOFFMANN A; NACHTIGAL JC. 2005. Propagação de plantas frutíferas. Brasília: Embrapa Informação Tecnológica, $221 \mathrm{p}$.

FERREIRA MGR; GONÇALVES EP. 2007. Estaquia e Crescimento Inicial de Crajiru. Revista Brasileira de Biociências 5: 363-365.

FRASSETO RG 2008. Enraizamento adventício de estacas de Sebastiania schottiana Müll. Arg. Santa Maria: UFSM.132p. (Tese doutorado).

HARTMANN HT; KESTER DE; DAVIES JUNIOR FT. 2002. Plant Propagation: principles and practicas. $7^{\text {th }}$ ed. New York: Englewood Clipps, 880p.

HOFFMANN A; FACHINELLO JC; SANTOS AM. 1995. Enraizamento de estacas de duas cultivares de mirtilo (Vaccinium ashei Reade) em diferentes substratos. Revista Brasileira de Agrociência 1: 7-11.

LIMA DM; SILVA CL; RITTER M; BIASI LA; ZANETTE F; ZUFFELLATO-RIBAS KC. 2008. Substratos e auxinas no enraizamento de estacas caulinares de espinheira-santa. Scientia Agraria 9: 85-89.

LIMA RLS; SIQUEIRA DL; WEBER OB; CAZETTA JO. 2006. Comprimento de estacas e parte do ramo na formação de mudas de aceroleira. Revista Brasileira de Fruticultura 28: 83-86.

LUZ PB; PAIVA PDO; LANDGRAF PRC. 2007. Influencia de diversos tipos de estacas e de substratos na propagação assexuada de hortênsia [Hydrangea macrophylla (Thunb.) Ser.] Ciência Agrotecnica 31: 699-703.

MAIA ACCM; SOARES FCC; MARTINS JUNIOR HB; BAPTISTA ER. 2001. Vitex agnus castus L: Um estudo etnobotânico e etnofarmacológico. Revista Virtual de Iniciação Acadêmica da UFPA 1: 1-15.

MAIA SSS; PINTO JEBP; SILVA FN; OLIVEIRA C. 2008. Enraizamento de Hyptis suaveolens (L.) Poit. (Lamiaceae) em função da posição da estaca no ramo. Revista Brasileira de Ciências Agrárias 3: 317-320.

MATOS FJA. 2002. Farmácias vivas: sistema de utilização de plantas medicinais projetado para pequenas comunidades. 4. ed. Fortaleza: Editora UFC, 267p.

PEREIRA AB; PASQUAL M; RIBEIRO LS; MENDES ANG; RESENDE E. 2002. Enraizamento de estacas de Coffea arabica L. em diferentes substratos. Ciência e Agrotecnologia 26: 741-748.

RIBEIRO JÚNIOR JI; MELO AFL. 2009. Guia prático para utilização do SAEG. Viçosa: Editora Independente, 287p.

SCHELLENBERG R. 2001.Treatment for the premenstrual syndrome with agnus castus fruit extract: prospective, randomised, placebo controlled study. Brithish Medical Journal 322: 134-137.

SCHMITZ JAK; SOUZA PVD; KÄMPF AN. 2002. Propriedades químicas e físicas de substratos de origem mineral e orgânica para o cultivo de mudas em recipientes. Ciência Rural 32: 937-944.

SILVA PNL; COSTA E; FERREIRAAFA; SILVA ACR; GOMES VA. 2010. Enraizamento de estacas de aceroleira: efeitos de recipientes e substratos. Revista Agrarian 3: 126-132.

SLIUTZ G; SPEISER P; SCHULTZAM; SPONA J; ZEILLINGER R. 1993. Agnus castus extracts inhibit prolactin secretion of rat pituitary cells. Hormone Metabolism Research 25: 253-255.

SOUSA PBL; AYALA-OSUNA JT; GOMES JE. 2005. Propagação vegetativa de Ocimum gratissimum L. em diferentes substratos. Revista Brasileira de Plantas Medicinais 8: 39-44.

VEAL L. 1998. Complementary therapy and infertility: an Icelandic perspective. ZFA 58: 228-231. 